See discussions, stats, and author profiles for this publication at: https://www.researchgate.net/publication/234014005

\title{
A Personalized Middleware for Ubiquitous mHealth Services
}

Conference Paper · October 2012

DOI: 10.1109/HealthCom.2012.6379465

CITATIONS

13

4 authors, including:

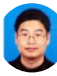

Zhanlin Ji

University of Limerick

88 PUBLICATIONS 616 CITATIONS

SEE PROFILE

2.) Mairtin S. O'Droma

University of Limerick

272 PUBLICATIONS 1,765 CITATIONS

SEE PROFILE

Some of the authors of this publication are also working on these related projects:

Project ACROSS View project

Project Service Recommendation Modelling View project
READS

178

Ivan Ganchev

Plovdiv University "Paisii Hilendarski"

299 PUBLICATIONS 1,696 CITATIONS

SEE PROFILE 


\title{
A Personalized Middleware for Ubiquitous mHealth Services
}

\author{
Zhanlin Ji ${ }^{1,2}$, Xueji Zhang ${ }^{1}$, Ivan Ganchev ${ }^{2}$, Máirtín O’Droma ${ }^{2}$ \\ ${ }^{1}$ Research Center for Bioengineering and Sensing Technology, University of Science and Technology Beijing, China \\ ${ }^{2}$ Telecommunications Research Centre, University of Limerick, Ireland \\ \{Zhanlin.ji; Zhangxueji\}@ustb.edu.cn; $\{$ Ivan.Ganchev; Mairtin.ODroma\}@ul.ie
}

\begin{abstract}
This paper presents a personalized middleware for mobile eHealth (mHealth) services for use in the Ubiquitous Consumer Wireless World (UCWW). The middleware was developed based on the ISO/IEEE 11073 personal health data (PHD) standards. It works as a multi-agent system (MAS) to provide intelligent collection of physiological data from medical sensors attached to human body, and subsequent sending of gathered data to a log data node by utilizing the Always Best Connected and best Served $(\mathrm{ABC} \& S)$ communication paradigm. A number of design issues associated with the middleware implementation are outlined.
\end{abstract}

Index Terms-ISO/IEEE 11073; Ubiquitous mHealth (uHealth); Body Area Network (BAN); Ubiquious Consumer Wireless World (UCWW); Multi-Agent System (MAS)

\section{INTRODUCTION}

The population in the world aged above 65 is expected to reach $12 \%$ in 2030 [1]. Healthcare costs will be a serious problem, especially for developing countries. The ISO/IEEE 11073 committee proposed a number of interoperability standards for personal health devices (PHD) [2]. In [3], an ultralow-power wireless sensor network solution was proposed. The authors stated that the design of a low-power sensor is very important as the battery lifetime is expected to increase only by $20 \%$ in the next ten years. Medical sensors were not mentioned in [3], but from research it is clear that reducing the power consumption is one of the main goals when designing wireless healthcare middleware. In [4], Libresoft reported on an open-source ISO/IEEE 11073 system with its own Bluetooth application program interface (API). The authors stated that it is not easy to develop a ubiquitous eHealth system operating in a cloud environment. In [5], Intel provided an evaluation software kit for the development of embedded devices, which are used to interact with medical devices. It is based on the Windows Mobile operating system, and the devices must be certified by Continua. In [6], a legacy healthcare device adaption hardware was designed for compatibility with the ISO/IEEE 11073 standards. The extra hardware, however, makes the system heavy. In [7], a ubiquitous mHealth (uHealth) system based on the ISO/IEEE 11073 standards was proposed, but the system seems not being able to provide a fully ubiquitous solution.

In this short paper, an ISO/IEEE 11073 based middleware, which works with the Ubiquitous Consumer Wireless World (UCWW) cloud in an intelligent way, is introduced. First, a software agent formats the collected physiological parameter values along with the user's location, medical device information, IP address etc, forming a web log and serializing it into a log server. Then a relevant cloud ant collects it by using the defined scheduling algorithms under the Always Best Connected and best Served (ABC\&S) communication paradigm [8], and receives back medical-care advices/instructions/statements which are presented to the user. The medical-care contents is personalized and customized to suit the mobile device and mobile user. The ISO/IEEE 11073 middleware is designed to operate 'anywhere-anytime-anyhow' [9]. With this advanced technology, it could be utilized by a mHealth system to provide medical services in a pervasive mode, thus making it a truly ubiquitous mHealth (uHealth) system.

\section{Personalised Healthcare Middleware in UHEALTh SyStem ARChitecture}

The uHealth system is designed as a tiered distributed system (Figure 1). In the sensor nodes tier, a number of

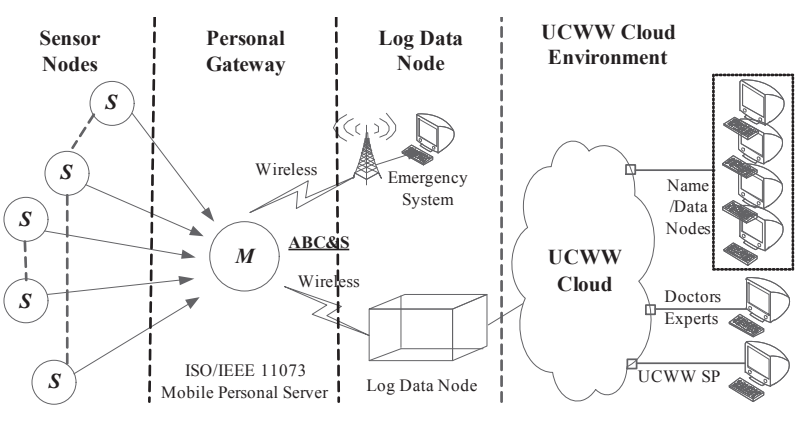

Fig. 1. The tiered uHealth System Architecture.

wired medical sensors (S) - such as pulse oximeter, heart rate monitor, blood pressure monitor, thermometer, weighting scale, and glucose meter, etc. They use Bluetooth or ZigBee for communication with the ISO/IEEE 11073 based middleware $(\mathrm{M})$ in the personal gateway tier. The gateway usually is a mobile phone, for example, a Google Android phone. An agent operating on the phone collects data from the sensor nodes and sends a personalized message to a log data node / emergency system. The log data node forwards the $\log$ to the UCWW cloud and receives back medicalcare advices, which are passed on to the user. In addition, an emergency system operates in real time to accept alarms 
about bad medical condition of the user. In the UCWW cloud environment, a set of Hadoop [10] data mining applications run for log data collecting, analyzing, processing and advising. Different users may receive different medical-care advices and statements based on their personal profiles and current physiological condition. Also, the cloud provides experts' and doctors' manual diagnosis.

Figure 2 shows the high-level view of the personalized healthcare middleware. The communication support layer is at the bottom of this architecture. It is provided by the Android operating system. The middle layer includes the ISO/IEEE 11073 PHD device specializations. The domain information model (DIM), service model, communication model, rule engine, and gateway agent operate at the top layer.

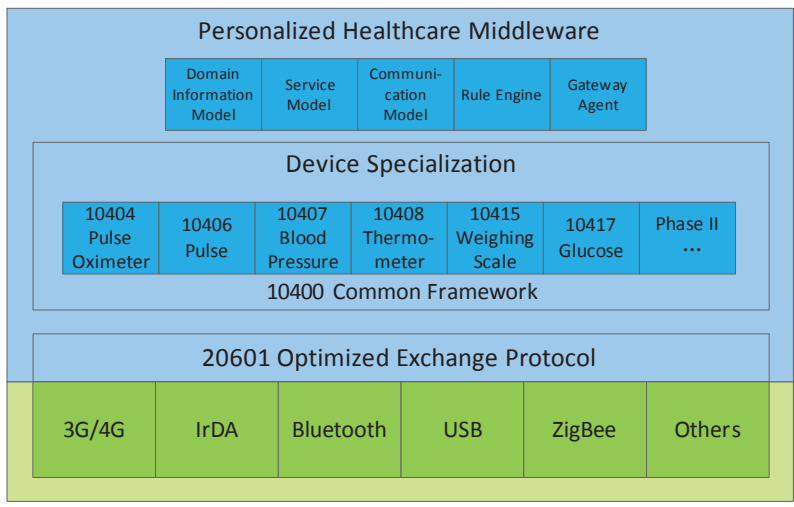

Fig. 2. The High-Level View of the Content Adaption Middleware

\section{MidDleWARE DESIGN}

The ISO/IEEE 11073 based middleware is designed as a key component of a uHealth system that enables users to avail of personalized health services using the $A B C \& S$ mode. Its main components are: the DIM, which defines the health device object, medical device system (MDS) and metrics; the service model, which defines the object access methods and event reporting; the communication model, which states the finite state machine (FSM) for the ISO/IEEE 11073 agent (sensor node) and the ISO/IEEE 11073 manager (middleware); the rule engine, which is used for separating the business logic from the middleware system; and the gateway agent, which acts as a bridge between the personal mobile server and the rest of the uHealth system. The middleware components are described in more detail in the following subsections.

\section{A. Domain Information Model (DIM):}

In the ISO/IEEE 11073 standards, the DIM includes several objects, e.g., for generating medical advices, collecting patient information, configuration etc (Figure 3). The medical device system (MDS) is a top-level object, which represents services and properties of the device. It includes a number of objects and device configuration parameters. The metric is a base object for representing the measurements and context data. It includes numeric, enumeration, real time sample array
(SA) and persistent metric (PM) segment objects. To exchange structured data between the device and middleware, the ASN.1-based medical device encoding rules (MDER) standard, including binary encoding rule (BER) and packet encoding rule (PER) coding schemes, was utilized.

\section{B. Service model:}

The service model is used to exchange data between sensor nodes and middleware. The message exchange and command execution mechanisms are provided.

\section{Communication Model:}

To enable multiple sensor nodes to communicate with the gateway, a FSM was used to define the states and sub-states used for connection, association, operation, and disconnection. Figure 3 shows the association control (ACSE) and the remote operation (ROSE) basic services in the communication model.

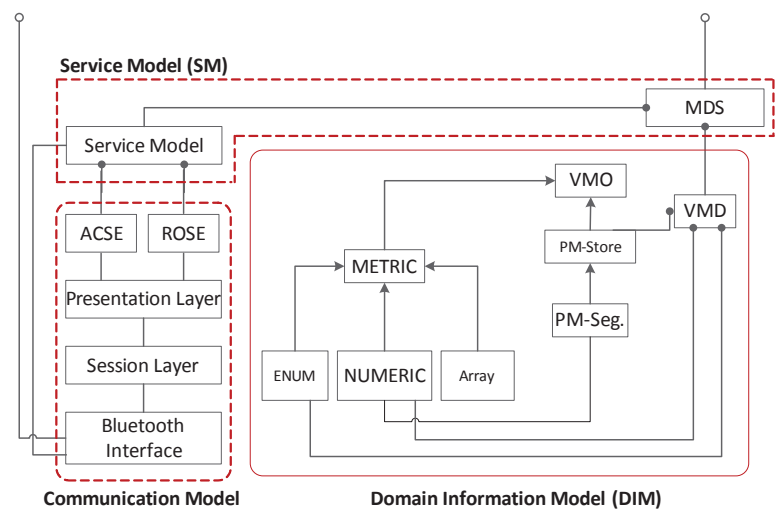

Fig. 3. The relationship between DIM, service model, and communication model in the middleware.

\section{Rule Engine:}

In order to be able to dynamically change the business logic and efficiently manage the uHealth system, a rule engine was selected for controlling the business behaviours, e.g. define/update personal profiles, location, log data node, etc. Other benefits of using this rule engine in the uHealth system are the separation of the business logic from the system implementation and the lack of necessity of changing the source code when the business logic is updated.

\section{E. Gateway Agent:}

An agent platform runs on the log data node. It provides agent management system (AMS), directory facilitator (DF) and message transport services. The gateway agent runs in mediator mode, i.e., the container is split into a front-end (running on the mobile personal server) and a back-end (running on the log data node). 


\section{F. Personalized:}

To achieve personalized middleware for uHealth system, a number of profiles are initialized on the 11073 manager side, including an identification/authentication profile, location profile, advertisement accept profile, rule profile, and history profile. The gateways agent reads/updates/manages all these profiles.

To save power based on the rule profile, the manager application operates in sleep mode. It is only on arrival of defined messages when it wakes up and pushes data to the $\log$ data node.

On the UCWW cloud side, a Device Description Repository (DDR) analyzes the client request header and provides the corresponding mobile device with compatible services.

\section{G. Security:}

As the mHealth service runs within a UCWW environment, security on the uHealth server side is provided by ThirdParty Authentication, Authorization and Accounting Service Providers (3P-AAA-SPs) [9], whereas on the device side it is provided by a smart consumer identity module (CIM) card [11].

\section{IMPLEMENTATION AND EXPERIMENTAL TESTING}

Designing the ISO/IEEE 11073 based healthcare middleware was successfully, quickly and efficiently done by using low complexity, low cost, open-source, academic-licensed APIs such as:

$J A D E$ - a MAS implemented in Java and developed by TILIB with the FIPA specifications;

OSS - Nokalva OSS ASN.1 Tools for Java API;

Drools - JBOSS with LGPL \& JESS (Sandia National Laboratories) rule-engine based expert systems;

$D B 4 O$ - a high performance, embeddable object-oriented database;

WURFL - a community effort focused on mobile device capabilities detection (AGPL license by ScientiaMobile);

Android - the designed middleware runs on a lightweight mobile personal server installed on an Android mobile device.

A pulse oximeter was used as a medical sensor device in the system testbed (Figure 4). For communication with middleware, an adapter was used to ensure compatibility. In future, a pure HTML5 compatible UI application will be developed to replace the Android application.

A pulse oximeter was used as a medical sensor device in this system testbed (Figure 6). For communication with middleware, an adapter was used to ensure compatibility. In future, a pure HTML5 compatible UI application will be developed to replace the Android application.

\section{CONCLUSION AND Future Work}

The design of a lightweight personalized ISO/IEEE 11073 based middleware has been presented in this paper. The middleware is written in Java and is easily integrated into an uHealth system. The middleware is controlled by a gateway agent to ensure personalization and intelligent usage. The

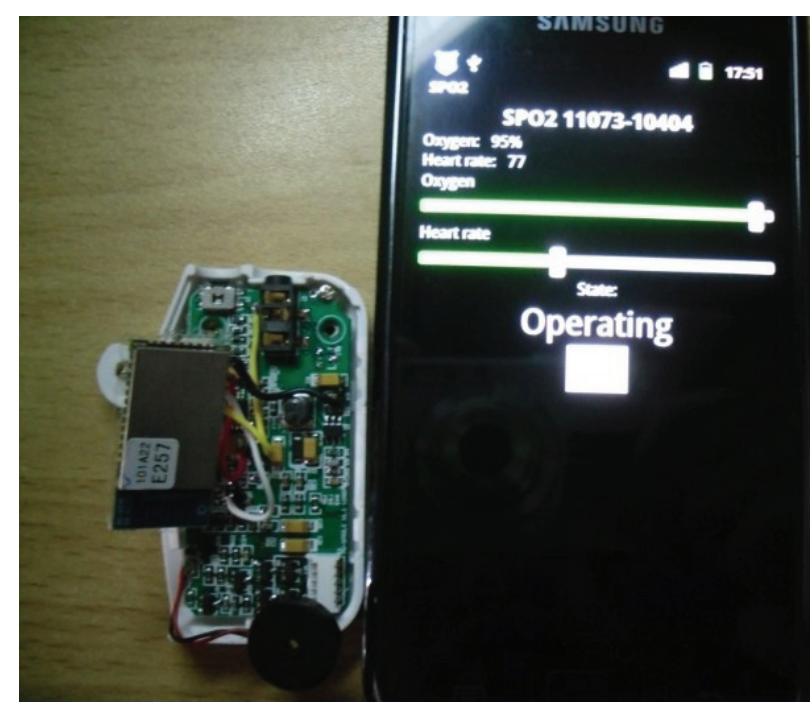

Fig. 4. The medical device in operation.

gateway runs as a mediator in the designed multi-agent system (MAS). A number of other components in the personalized ISO/IEEE 11073 based middleware have been also detailed. With the proposed middleware, the uHealth providers can offer more comfortable cloud-based medical services to their users.

The next step of our work includes integrating the IEEE 11073 based electrocardiography (ECG) and blood pressure sensors into the system. The performance of the FSM will be tested as well. On the UCWW cloud side, corresponding data mining algorithms will be developed for doctors and medical service providers to push only those messages (medical advices/ads) to end users that are of interest to them.

\section{REFERENCES}

[1] Y. He, W. Zhu, L. Guan, "Optimal Resource Allocation for Pervasive Health Monitoring Systems with Body Sensor Networks", IEEE Transactions on Mobile Computing Vol. 10, No. 11, Pp. 1558-1575, 2011.

[2] ISO/IEEE 11073 Committee. Available at: http://www.ieee1073.org.

[3] C. C. Enz, A. El-Hoiydi, J. D. Decotignie, and V. Peiris, "WiseNET: An ultralow-power wireless sensor network solution," IEEE Computer, Vol. 37, No. 8, Pp. 62-70, Aug. 2004.

[4] GSyC/Libresoft. OpenHealth project. http://openhealth.morfeoproject.org/.

[5] Intel. Evaluation kit with IEEE 11073 Continua-certified software stack for medical applications. http://www.intel.com/p/en_US/embedded /applicationsmedical/evaluation-kit/overview.

[6] C.-Y. Park, J.-H. Lim, and S. Park. "ISO/IEEE 11073 PHD standardization of legacy healthcare devices for home healthcare services". 2011 IEEE International Conference on Consumer Electronics (ICCE), USA, Pp. 547-548, Jan. 2011.

[7] J. Nam, W. Seo, J. Bae, Y. Cho, "Design and Development of a u-Health System Based on the ISO/IEEE 11073 PHD Standards", 17th Asia-Pacific Conference on Communications (APCC), Malaysia, Pp. x1-x5, Oct. 2011.

[8] Z. Ji, I. Ganchev, and M. O'Droma, "An iWBC consumer application for 'always best connected and best served': design and implementation," IEEE Transactions on Consumer Electronics, Vol. 57, No. 2, Pp. 462470, May 2011.

[9] M. O'Droma, and I. Ganchev, "Toward a ubiquitous consumer wireless world," IEEE Wireless Communications, Vol. 14, No. 1, Pp. 52-63, 2007.

[10] White T: Hadoop: The Definitive Guide. Sebastopol: O'Reilly Media; 2009.

[11] M. O'Droma, and I. Ganchev. "The Creation of a Ubiquitous Consumer Wireless World through Strategic ITU-T Standardization". IEEE Communications Magazine. Vol. 48, No. 10, Pp. 158-165, 2010. 